Pacific Journal of Mathematics

THE HAUSDORFF MEANS OF DOUBLE FOURIER SERIES 


\title{
THE HAUSDORFF MEANS OF DOUBLE FOURIER SERIES AND THE PRINCIPLE OF LOCALIZATION
}

\author{
FRED Ustina
}

\begin{abstract}
In the two dimensional case, as in the one dimensional case, the Hausdorff summability method is generated by a Hausdorff weight function. In this paper, we investigate the conditions which must be imposed on this weight function in order that the resulting means of a double Fourier series will display the principle of localization.
\end{abstract}

In this article we examine the conditions under which the Hausdorff means of double Fourier series exhibit the principle of localization. As is well known, these means are a generalization of a number of other well known means, including those of Cesáro and Euler. Our results are summarized in Theorems 1 to 4, together with the appropriate corollaries.

Let $[c, d ; a, b]$ denote a rectangle with vertices at $(a, b),(a, d),(c, b)$ and $(c, d), a \leqq c, b \leqq d$. For $0<\delta<\pi$, let $R(\delta)=[\delta, \delta ;-\delta,-\delta]$, $N(\delta)=[\pi, \delta ;-\pi,-\delta] \cup[\delta, \pi ;-\delta,-\pi], C(\delta)=[\pi, \pi ;-\pi,-\pi] \sim N(\delta)$, and $E(\delta)=N(\delta) \sim R(\delta)$. For $0<\tau \leqq 1 / 2$, let $\Delta(\tau)=[1-\tau, 1-\tau ; \tau, \tau]$, and let $\theta(\tau)=[1,1 ; 0,0] \sim \Delta(\tau)$. Then $N(\delta)$ is a cross-neighborhood of the origin, $E(\delta)$ is the deleted cross-neighborhood, and $\theta(\tau)$ is the $\tau$-neighborhood of the boundary of the unit square $[1,1 ; 0,0]$.

Let $f(x, y)$ be a $2 \pi$-periodic function, Lebesgue integrable in the period square, and let $\left\{s_{m n}(x, y)\right\}$ be the corresponding sequence of partial sums of the Fourier series of $f(x, y)$. In the sequel we relate all such calculations to the origin, so that we will be examining the sequence $\left\{s_{m n}(0,0)\right\}$, which we denote simply by $\left\{s_{m n}\right\}$. As is easily shown,

$$
\begin{aligned}
s_{m n} & =\frac{1}{4 \pi^{2}} \int_{-\pi,-\pi}^{\pi, \pi} f(s, t) \frac{\sin (m+1 / 2) s}{\sin s / 2} \frac{\sin (n+1 / 2) t}{\sin t / 2} d s d t \\
& =\frac{1}{4 \pi^{2}}\left\{\int_{R(\delta)}+\int_{E(\delta)}+\int_{C(\delta)}\right\} \\
& =r_{m n}+e_{m n}+c_{m n} .
\end{aligned}
$$

Now suppose that a regular linear summability method $H$ ([6], Vol. 1, p. 74) is applied to the sequence $\left\{s_{m n}\right\}$ and let $\left\{h_{m n}\right\}$ denote the corresponding sequence of transforms under the method $H$. Then

$$
\begin{aligned}
h_{m n} & =H\left\{s_{m n}\right\} \\
& =H\left\{r_{m n}\right\}+H\left\{e_{m n}\right\}+H\left\{c_{m n}\right\} \\
& =\alpha_{m n}+\beta_{m n}+\gamma_{m n} .
\end{aligned}
$$


We will say that the principle of localization holds for the sequence $\left\{s_{m n}\right\}$ if for arbitrary, fixed $\delta, s_{m n}=r_{m n}+o(1), m, n \rightarrow \infty$, and that it holds restrictedly if $s_{m n}=r_{m n}+o(1), m, n \rightarrow \infty$, under certain specified restrictions on the subscripts $m$ and $n$. Similarly, we will say that the principle of localization holds for the sequence $\left\{h_{m n}\right\}$ if $h_{m n}=\alpha_{m n}+o(1), m, n \rightarrow \infty$, and that it holds restrictedly if

$$
h_{m n}=\alpha_{m n}+o(1), m, n \rightarrow \infty,
$$

under certain specified restrictions on the subscripts $m$ and $n$.

It follows that a necessary and sufficient condition for the principle of localization to hold in the first case is that

$$
e_{m n}+c_{m n}=o(1), m, n \rightarrow \infty,
$$

and in the second case the necessary and sufficient condition is that $\beta_{m n}+\gamma_{m n}=o(1), m, n \rightarrow \infty$. Since $f(x, y)$ is assumed to be Lebesgue integrable, the sequence $\left\{c_{m n}\right\}$ is a null sequence. Since the method $H$ is assumed to be regular, it follows that the sequence $\left\{\gamma_{m n}\right\}$ is also a null sequence. Thus to prove that the principle of localization holds for an arbitrary, Lebesgue integrable function, it is necessary and sufficient to prove that, for an arbitrary fixed $\delta, \delta>0, e_{m n}=o(1)$ and $\beta_{m n}=o(1), m, n \rightarrow \infty$, in the respective cases.

Now let $H$ be a regular Hausdorff summability method. This method has been investigated in detail by Hallenbach [2]. From the foregoing, it follows that to prove that the principle of localization holds for this method, it is necessary and sufficient to prove that the sequence $\left\{\beta_{m n}\right\}$ is a null sequence, where

$$
\beta_{m n}=\sum_{0,0}^{m, n}\left(\begin{array}{c}
m \\
k
\end{array}\right)\left(\begin{array}{c}
n \\
l
\end{array}\right) e_{k l} \int_{0,0}^{1,1} u^{k}(1-u)^{m-k} v^{l}(1-v)^{n-l} d g(u, v),
$$

where the Hausdorif weight function $g(u, v)$ is of bounded variation in the unit square $[1,1 ; 0,0], g(u, 0)=g\left(u, 0^{+}\right)=g(0, v)=g\left(0^{+}, v\right)=0$, and $g(1,1)=1$.

We will say that $g(u, v)$ satisfies a Lipschitz condition of order one and Lipschitz constant $M$ on a region $R \subset[1,1 ; 0,0]$ if $g(u, v)$ is continuous on $R$ and if

$$
\left|g\left(u^{\prime \prime}, v^{\prime \prime}\right)-g\left(u^{\prime}, v^{\prime \prime}\right)-g\left(u^{\prime \prime}, v^{\prime}\right)+g\left(u^{\prime}, v^{\prime}\right)\right| \leqq M\left|\left(u^{\prime \prime}-u^{\prime}\right)\left(v^{\prime \prime}-v^{\prime}\right)\right|
$$

whenever the rectangle $\left[u^{\prime \prime}, v^{\prime \prime} ; u^{\prime}, v^{\prime}\right]$ contains only points of $R$. It is easily seen that in such a case, the absolute value of the measure $d g(u, v)$ is majorized by $M d u d v$ on $R$.

Summary of the main results. We assume that $g(u, v)$ is a regular Hausdorff weight function, so that the corresponding Hausdorff 
method is regular. From (3), we have

$$
\text { (4) } \begin{aligned}
\beta_{m n} & =\sum_{0,0}^{m, n}\left(\begin{array}{c}
m \\
k
\end{array}\right)\left(\begin{array}{c}
n \\
l
\end{array}\right) e_{k l}\left\{\int_{\Lambda(\tau)}+\int_{;(\tau)}\right\} u^{k}(1-u)^{m-k} v^{l}(1-v)^{n-l} d g(u, v) \\
& =\delta_{m n}+\theta_{m n} .
\end{aligned}
$$

THeOREM 1. If a $2 \pi$-periodic function is Lebesgue integrable in the period square, then $\delta_{m n}=o(1), m, n \rightarrow \infty$, provided that for some fixed but otherwise arbitrary $\varepsilon, 0<\varepsilon \leqq 1$, and $\kappa=\tau(1-\tau) \delta^{2} / 12$, we have that $m \leqq \varepsilon^{-1} e^{\kappa n}$ and $n \leqq \varepsilon^{-1} e^{\kappa m}$, a fortiori provided that

$$
\varepsilon m^{-k} \leqq m / n \leqq \varepsilon^{-1} n^{k}
$$

for an arbitrary, fixed positive integer $k$, as $m, n \rightarrow \infty$.

COROLLARY 1. If $\left\{h_{m n}\right\}$ is a sequence of regular Hausdorff transforms of the sequence $\left\{s_{m n}\right\}$, corresponding to a $2 \pi$-periodic function which is Lebesgue integrable on the period square, then the question of whether the sequence $\left\{h_{m n}: \varepsilon m^{-k} \leqq m / n \leqq \varepsilon^{-1} n^{k}\right\}$ exhibits the prinsiple of localization depends entirely on the behavior of the Hausdorff weight function in an arbitrarily small $\tau$-neighborhood of the boundary of the unit square $[1,1 ; 0,0]$.

Corollary 2. If, in Corollary 1 , the sequence $\left\{h_{m n}\right\}$ corresponds to a Hausdorff weight function $g(u, v)$ such that for some $\tau>0$, the measure $d g(u, v)$ is identically zero in a $\tau$-neighborhood of the boundary of the unit square, then the sequence $\left\{h_{m n}: \varepsilon m^{-k} \leqq m / n \leqq \varepsilon^{-1} n^{k}\right\}$ exhibits the principle of localization. In partisular, if $0<\alpha<1$ and $0<\beta<1$, and if $\left\{\varepsilon_{m n}\right\}$ is the sequence of the Euler $(\varepsilon ; \alpha, \beta)$ transforms of the sequence $\left\{s_{m n}\right\}$, corresponding to a $2 \pi$-periodic function Lebesgue integrable on the period square, then the sequence

$$
\left\{\varepsilon_{m n}: \varepsilon m^{-k} \leqq m / n \leqq \varepsilon^{-1} n^{k}\right\}
$$

exhibits the principle of localization.

Corollary 1 is an obvious consequence of Theorem 1. This is also the case with the first part of Corollary 2. The second part of Corollary 2 then follows by observing that the Euler $(\varepsilon ; \alpha, \beta)$ means are Hausdorff means corresponding to a function $g(u, v)$ such that the measure $d g(u, v)$ is identically zero except at the point $(\alpha, \beta)$, where $d g(u, v)=1$.

THEOREM 2. Let $\sigma$ be any subset of $\theta(\tau)$ such that on every simply connested subset of $\sigma$, the Hausdorff weight function $g(u, v)$ satisfies a Lipschitz condition of order one with Lipschitz constant $M$. Let 


$$
\sigma_{m n}=\sum_{0,0}^{m, n}\left(\begin{array}{c}
m \\
k
\end{array}\right)\left(\begin{array}{c}
n \\
l
\end{array}\right) e_{k l} \int_{\sigma} u^{k}(1-u)^{m-k} v^{l}(1-v)^{n-l} d g(u, v) \text {. }
$$

Then we have

(a) if $f(x, y) \in L_{p}(N(\delta)), 1<p<\infty$, then $\sigma_{m n}=o(1), m, n \rightarrow \infty$, $\varepsilon m^{-\lambda} \leqq m / n \leqq \varepsilon^{-1} n^{\lambda}$, where $\varepsilon$ and $\lambda$ are fixed but arbitrary, $0<\varepsilon \leqq 1$, and $0<\lambda<p-1$.

(b) if $f(x, y) \in B(N(\delta))$, then $\sigma_{m n}=o(1), m, n \rightarrow \infty, m \leqq \varepsilon^{-1} e^{n^{\Lambda}}$, $n \leqq \varepsilon^{-1} e^{m^{\lambda}}$, where now $\varepsilon$ and $\lambda$ are fixed, $0<\varepsilon \leqq 1$ and $0<\lambda<1$.

In particular, the contribution to the mnth term of the sequence $\left\{\beta_{m n}\right\}$, due to integration with respect to $g(u, v)$ over any subset of the unit square on which $g(u, v)$ satisfies a Lipschitz condition of order one with Lipschitz constant $M$, is o(1), $m, n \rightarrow \infty, \varepsilon m^{-\lambda} \leqq m / n \leqq \varepsilon^{-1} n^{\lambda}$, $0<\lambda<p-1$, if $f(x, y) \in L_{p}(N(\delta))$; and this contribution is

$$
o(1), m, n \rightarrow \infty, m \leqq \varepsilon^{-1} e^{n^{\lambda}}, n \leqq \varepsilon^{-1} e^{m^{\lambda}}, 0<\lambda<1,
$$

if $f(x, y) \in B(N(\delta))$.

Corollary. Let $\left\{c_{m n}\right\}$ denote the sequence of the Cesáro $(C ; \alpha, \beta)$ means of $\left\{s_{m n}\right\}$, corresponding to a $2 \pi$-periodic function $f(x, y)$. If $1 \leqq \alpha, \beta$, and if $f(x, y) \in B(N(\delta))$, then for arbitrary fixed $\varepsilon, \lambda, 0<\varepsilon \leqq 1$, $0<\lambda<1$, the sequence $\left\{c_{m n}: m \leqq \varepsilon^{-1} e^{n^{\lambda}}, n \leqq \varepsilon^{-1} e^{m^{\lambda}}\right\}$ exhibits the principle of losalization. If $1 \leqq \alpha, \beta$, and if $f(x, y) \in L_{p}(N(\delta)), 1<p<\infty$, then for arbitrary, fixed $\varepsilon, \lambda, 0<\varepsilon \leqq 1,0<\lambda<p-1$, the sequence

$$
\left\{c_{m n} ; \varepsilon m^{-\lambda} \leqq m / n \leqq \varepsilon^{-1} n^{\lambda}\right\}
$$

exhibits the principle of localization.

The corollary follows from the theorem by taking $\sigma=[1,1 ; 0,0]$ and observing that the $(C ; \alpha, \beta)$ means are Hausdorff means corresponding to the function $g(u, v)=\left\{1-(1-u)^{\alpha}\right\}\left\{1-(1-v)^{\beta}\right\}$. Then $|d g(u, v)|=\left|\alpha \beta(1-u)^{\alpha-1}(1-v)^{\hat{\beta}-1}\right| d u d v \leqq M d u d v$ for some constant $M \geqq \alpha \beta$ if $1 \leqq \alpha, \beta$.

Since it is already known ([6], Vol. II, p. 304) that for bounded $f(x, y)$, the sequence $\left\{c_{m n}\right\}$ exhibits the principle of localization without the restrictions imposed on the subscripts $m, n$ in the above corollary, it would seem that our estimates in Theorem 2 can be improved or a new proof devised to give a better result. Attempts to achieve this have been unsuccessful so far.

THEOREM 3. Suppose that the Hausdorff weight function $g(u, v)$ is discontinuous along finitely many lines

$$
\omega=\left\{u=u_{i}, i=1,2, \cdots, k ; v=v_{j}, j=1,2, \cdots, l\right\},
$$


such that for some $\tau>0, \tau \leqq u_{i} \leqq 1-\tau, \tau \leqq v_{j} \leqq 1-\tau$ for all $i, j$. Let $g_{i}(v)=g\left(u_{i}^{+}, v\right)-g\left(u_{i}^{-}, v\right)$ and $g_{j}(u)=g\left(u, v_{j}^{+}\right)-g\left(u, v_{j}^{-}\right)$, and suppose that the functions $\left\{g_{i}(v), g_{j}(u)\right\}$ all satisfy a Lipschitz condition of order one and Lipschitz constant $M$. Let

$$
\omega_{m n}=\sum_{0,0}^{m, n}\left(\begin{array}{c}
m \\
k
\end{array}\right)\left(\begin{array}{c}
n \\
l
\end{array}\right) e_{k l} \int_{\omega} u^{k}(1-u)^{m-k} v^{l}(1-v)^{n-l} d g(u, v)
$$

be the contribution to $\beta_{m n}$ due to integration with respect to $g(u, v)$ along these lines. Then we have

(a) if $f(x, y) \in L[\pi, \pi ;-\pi,-\pi]$, then $\omega_{m n}=o(1), m, n \rightarrow \infty$, provided $\varepsilon \leqq m / n \leqq \varepsilon^{-1}$, where $0<\varepsilon \leqq 1$ is fixed but otherwise arbitrary.

(b) if $f(x, y) \in L_{p}(N(\delta))$, then

$$
\omega_{m n}=o(1), m, n \rightarrow \infty, \varepsilon m^{-\lambda} \leqq m / n \leqq \varepsilon^{-1} n^{\lambda},
$$

where $0<\varepsilon \leqq 1$, and $0<\lambda<p-1$ are fixed but otherwise arbitrary.

(c) if $f(x, y) \in B(N(\delta))$, then $\omega_{m n}=o(1), m, n \rightarrow \infty, m \leqq \varepsilon^{-1} e^{n^{\lambda}}$, $n \leqq \varepsilon^{-1} e^{m^{\lambda}}$, where $\varepsilon$ is as before, and $\lambda$ is again fixed, $0<\lambda<1$.

From Theorems 1,2 and 3 , a number of general statements pertaining to the principle of localization for the sequence $\left\{h_{m n}\right\}$ of regular Hausdorfi transforms of the sequence $\left\{s_{m n}\right\}$ can be made immediately. We give one example. From Theorems 1 and 3, we get

THEOREM 4. Suppose that $f(x, y) \in L[\pi, \pi ;-\pi,-\pi]$, that $f(x, y)$ is periodic of period $2 \pi$, and that $\left\{s_{m n}\right\}$ is the sequence of partial sums of the Fourier series of $f(x, y)$. If the Hausdorff weight function $g(u, v)$ is such that for some $\tau>0$ the measure $d g(u, v)$ is identically zero in the $\tau$-neighborhood of the boundary of the unit square, except along finitely many lines $u=u_{i}, v=v_{j}, \tau \leqq u_{i} \leqq 1-\tau, \tau \leqq v_{j} \leqq 1-\tau$ for all $i, j$, along which the difference functions $g_{i}(v), g_{j}(u)$, as defined in Theorem 3, all satisfy a Lipschitz condition of order one and Lipschitz constant $M$, then the corresponding sequence of Hausdorff transforms $\left\{h_{m n}\right\}$ exhibits the principle of localization restrictedly, with the restriction that for arbitrary fixed $\varepsilon, 0<\varepsilon \leqq 1$, we have

$$
\varepsilon \leqq m / n \leqq \varepsilon^{-1}
$$

Preliminary Lemmas. We collect here a few lemmas to facilitate the proofs of our results.

LEMMA 1. Let

$$
\begin{aligned}
& \rho_{1} \sin \alpha=u \sin s, \rho_{1} \cos \alpha=1-u+u \cos s \\
& \rho_{2} \sin \beta=v \sin t, \rho_{2} \cos \beta=1-v+v \cos t .
\end{aligned}
$$


Then

(7)

$$
\begin{gathered}
\sum_{0,0}^{m, n}\left(\begin{array}{c}
m \\
k
\end{array}\right)\left(\begin{array}{c}
n \\
l
\end{array}\right) \frac{\sin (k+1 / 2) s}{\sin s / 2} \frac{\sin (l+1 / 2) t}{\sin t / 2} u^{k}(1-u)^{m-k} v^{l}(1-v)^{n-l} \\
=\rho_{1}^{m} \rho_{2}^{n} \phi(m ; \alpha, s) \phi(n ; \beta, t)
\end{gathered}
$$

where

$$
\begin{aligned}
& \dot{\phi}(m ; \alpha, s)=\sin m \alpha \cot s / 2+\cos m \alpha \\
& \dot{\phi}(n ; \beta, t)=\sin n \beta \cot t / 2+\cos n \beta .
\end{aligned}
$$

Proof. The proof is essentially due to Szász [3, p. 443].

$$
\begin{aligned}
& \sum_{0}^{m}\left(\begin{array}{c}
m \\
k
\end{array}\right) \sin (k+1 / 2) s u^{k}(1-u)^{m-k} \\
= & \operatorname{Im}\left\{\left(1-u+u e^{i s}\right)^{m} e^{i s / 2}\right\} \\
= & \operatorname{Im}\left\{\left\{\rho_{1}(\cos \alpha+i \sin \alpha)\right\}^{m}(\cos s / 2+i \sin s / 2)\right\} \\
= & \rho_{1}^{m} \operatorname{Im}\{(\cos m \alpha+i \sin m \alpha)(\cos s / 2+i \sin s / 2)\} \\
= & \rho_{1}^{m}(\sin m \alpha \cos s / 2+\cos m \alpha \sin s / 2) .
\end{aligned}
$$

Dividing through by $\sin s / 2$ and noting that (7) is a product of two sums of the type (8) completes the proof.

Lemma 2. For small values of $t$, say $|t| \leqq \delta$, and $0 \leqq v \leqq 1$, (9) $\sin n \beta=\sin n t v+2 \cos n\left(t v+\frac{1}{2} r v(1-v) t^{3}\right) \sin n r v(1-v) t^{3} / 2$, where $|r|=|r(t, v)| \leqq \delta^{\prime}$.

Proof. This result is due to Szász [3, p. 449], taking into account that $\sin (-x)=-\sin x$.

Lemma 3. $|\phi(m ; \alpha, s)| \leqq \cot s / 2+1,0<s \leqq \pi$, so that for $0<\delta \leqq s \leqq \pi,|\phi(m ; \alpha, s)| \leqq \gamma<\infty$ uniformly in $m, \alpha$ and $s$.

Proof. The lemma is obvious.

LEMma 4.

$$
\dot{\phi}(n ; \beta, t)=2 \frac{\sin n \beta}{t}+\psi^{\prime}(n ; t, v)
$$

where ' $^{\prime}(n ; t, v)$ is bounded absolutely and uniformly, $0 \leqq v \leqq 1$, $0<|t| \leqq \pi, n=1,2, \cdots$.

Proof. From Lemma 1, we have 


$$
\begin{aligned}
\phi(n ; \beta, t) & =2 \frac{\sin n \beta}{t}+\sin n \beta\{\cot t / 2-2 / t\}+\cos n \beta \\
& =2 \frac{\sin n \beta}{t}+\psi^{\prime}(n ; t, v) .
\end{aligned}
$$

LemmA 5. For small values of $t$, say $|t| \leqq \delta$, and $0 \leqq v \leqq 1$,

$$
\frac{\sin n \beta}{t}=\frac{\sin n t v}{t}+\psi^{\prime \prime}(n ; t, v)\left\{n v(1-v) t^{2} / 12\right\},
$$

where $\left|\psi^{\prime \prime}(n ; t, v)\right| \leqq 12 \delta^{\prime}$.

Proof. The lemma follows immediately from Lemma 2 on division by $t$ since $|\cos x| \leqq 1$ and $|\sin x| \leqq|x|$. Thus the second term on the right in (9) is bounded absolutely by the absolute value of $n r v(1-v) t^{3}$. Thus

$$
\sin n \beta=\sin n t v+\psi^{\prime \prime}(n ; t, v)\left\{n v(1-v) t^{3} / 12\right\},
$$

where $\left|\psi^{\prime \prime}(n ; t, v)\right| \leqq|12 r(t, v)| \leqq 12 \delta^{\prime}$.

Lemma 6 . For $|s| \leqq \pi$ and $0 \leqq u \leqq 1$,

$$
\rho_{1}^{m} \leqq e^{-m u(1-u) s^{2} / 12} \text {. }
$$

Proof. From the definition in Lemma 1, we have

$$
\begin{aligned}
\rho_{1}^{2} & =1-2 u(1-u)(1-\cos s) \\
& =1-2 u(1-u)\left\{\left(s^{2} / 2 !-s^{4} / 4 !\right)+\left(s^{6} / 6 !-s^{8} / 8 !\right)+\cdots\right\} \\
& \leqq 1-2 u(1-u) s^{2}\left(1 / 2 !-s^{2} / 4 !\right) \\
& \leqq 1-u / 6(1-u) s^{2} \\
& \leqq e^{-u / 6(1-u) s^{2}}
\end{aligned}
$$

since under the restrictions on $s$ and $u, u(1-u)$, and each of the paired terms in $s$, is nonnegative, and $1 / 2 !-s^{2} / 4 !>1 / 12$.

REMARK. It is clear that under similar restrictions on $t$ and $v$, we have

$$
\rho_{2}^{n} \leqq e^{-n v(1-v) t^{2} / 12}
$$

Lemma 7. In the interval $0 \leqq v \leqq 1$ and $|t| \leqq \delta$,

$$
\rho_{2}^{n} \phi(n ; \beta, t)=2 \rho_{2}^{n} \frac{\sin n t v}{t}+\psi(n ; t, v)
$$

where $\psi(n ; t, v)$ is bounded absolutely and uniformly in $n, t$ and $v$, 
$\operatorname{say}|\psi(n ; t, v)| \leqq \delta^{\prime \prime}$

\section{Proof. By Lemmas 4 and 5,}

(10) $\phi(n ; \beta, t)=2 \frac{\sin n t v}{t}+2 \psi^{\prime \prime}(n ; t, v) n v(1-v) t^{2} / 12+\psi^{\prime}(n ; t, v)$.

Then

$$
\begin{aligned}
& \rho_{2}^{n} \phi(n ; \beta, t)=2 \rho_{2}^{n} \frac{\sin n t v}{t}+2 \psi^{\prime \prime}(n ; t, v) \rho_{2}^{n}(n / 12) v(1-v) t^{2} \\
&+\rho_{2}^{n} \psi^{\prime}(n ; t, v) \\
&=2 \rho_{2}^{n} \frac{\sin n t v}{t}+\psi(n ; t, v)
\end{aligned}
$$

where $\psi(n ; t, v)$ is bounded absolutely and uniformly, since $\psi^{\prime}(n ; t, v)$ and $\psi^{\prime \prime}(n ; t, v)$ are so bounded, and

$$
\rho_{2}^{n}(n / 12) v(1-v) t^{2} \leqq(n / 12) v(1-v) t^{2} e^{-n v(1-v) t^{2} / 12} \leqq e^{-1}
$$

for all values of $n, v$ and $t$, since $z e^{-z} \leqq e^{-1}$ for $z \geqq 0$.

Lemma 8. For $n$ and $k$ large enough, $0 \leqq v \leqq 1$ and $|t| \leqq \delta$,

$$
\left|\rho_{2}^{n} \phi(n ; \beta, t)\right| \leqq 4 n
$$

and

$$
|\dot{\phi}(n ; \beta, t)| \leqq 4 k n
$$

Proof. The first part follows immediately from Lemma 7 since $\rho_{2}^{n} \leqq 1, \psi(n ; t, v)$ is bounded, and $|\sin n t v / t| \leqq n v \leqq n$. The second part follows from $(10)$ since $\psi^{\prime}(n ; t, v)$ and $\psi^{\prime \prime}(n ; t, v)$ are bounded, and $n v(1-v) t^{2} / 12<n$ for $0 \leqq t \leqq \pi$.

LEMMA 9. If $K$ and $\kappa$ are any fixed, positive numbers, however, large or small, then

$$
K\left\{m e^{-\kappa n}+n e^{-\kappa m}\right)=o(1), m, n \rightarrow \infty,
$$

provided that for some fixed $\lambda, 0<\lambda<\kappa$, and fixed $\varepsilon, 0<\varepsilon \leqq 1$, we have $m \leqq \varepsilon^{-1} e^{\lambda n}$ and $n \leqq \varepsilon^{-1} e^{\lambda m}$ as $m, n \rightarrow \infty$, a fortiori, provided that $\varepsilon \leqq m / n \leqq \varepsilon^{-1}$ as $m, n \rightarrow \infty$.

Proof. We prove only that under these conditions, $K m e^{-\kappa n}=o(1)$, $m, n \rightarrow \infty$, the proof of the other part being the same. But then

$$
\begin{aligned}
K m e^{-\kappa n} & \leqq K \varepsilon^{-1} e^{-(\kappa-\wedge) n} \\
& \rightarrow 0, n \rightarrow \infty .
\end{aligned}
$$


Lemma 10. For $0<\delta \leqq s \leqq \pi$,

$$
\int_{0}^{1} \rho_{1}^{m} d u<c m^{-1}
$$

where $c=48 / \delta^{2}$.

Proof. By Lemma 6,

$$
\begin{aligned}
\int_{0}^{1} \rho_{1}^{m} d u & \leqq \int_{0}^{1} e^{-m u(1-u) s^{2} / 12} d u \\
& \leqq \int_{0}^{1} e^{-m u(1-u) \delta^{2} / 12} d u .
\end{aligned}
$$

Now since the integrand on the right is symmetric in $u$ and $1-u$, and $u(1-u) \geqq u / 2,0 \leqq u \leqq 1 / 2$, we get

$$
\begin{aligned}
\int_{0}^{1} \rho_{1}^{m} d u \leqq & \int_{0}^{1 / 2} e^{-m u(1-u) \delta^{2} / 12} d u \\
\leqq & 2 \int_{0}^{1 / 2} e^{-m u \tilde{\delta}^{2} / 24} d u \\
& <48 / m \delta^{2}=c m^{-1}
\end{aligned}
$$

Lemma 11. For $0 \leqq v \leqq 1$ and $0<\delta \leqq \pi$,

$$
\int_{0}^{o}\left|\frac{\sin n t v}{t}\right| d t<2 \log n, n \geqq 10 \text {. }
$$

Proof. If $v=0$, the lemma is obvious. If $v \neq 0$, set $n t v=t^{\prime}$. Then

$$
\begin{aligned}
\int_{0}^{\delta}\left|\frac{\sin n t v}{t}\right| d t & =\int_{0}^{n v \delta}\left|\frac{\sin t}{t}\right| d t \\
\leqq & \int_{0}^{\pi n}\left|\frac{\sin t}{t}\right| d t \\
& <\int_{0}^{1} \frac{\sin t}{t} d t+\int_{1}^{\pi n} \frac{d t}{t} \\
& <\{1+\log \pi+\log n\} \\
& <2 \log n, n \geqq 10 .
\end{aligned}
$$

Lemma 12. For $0 \leqq v \leqq 1, \quad 0<\delta \leqq \pi$ and $1 / p+1 / q=1$, $1<p<\infty$, we have

$$
\left\{\int_{0}^{o}\left|\frac{\sin n t v}{t}\right|^{q} d t\right\}^{1 / q}<2 n^{1 / p} \log n, n \geqq 10 .
$$

Proof. Again, if $v=0$, the result is obvious. Otherwise set 
$n t v=t^{\prime}$. Then

$$
\begin{aligned}
\left\{\int_{0}^{o}\left|\frac{\sin n t v}{t}\right|^{q} d t\right\}^{1 / q} & =\left\{\int_{0}^{n v \delta}\left|n v \frac{\sin t}{t}\right|^{q} \frac{d t}{n v}\right\}^{1 / q} \\
\leqq & \left\{(n v)^{q-1} \int_{0}^{\pi n}\left|\frac{\sin t}{t}\right|^{q} d t\right\}^{1 / q} \\
\leqq & n^{1 / p}\left\{\int_{0}^{\pi n}\left|\frac{\sin t}{t}\right| d t\right\}^{1 / q} \\
& <n^{1 / p}\{1+\log \pi+\log n\}^{1 / q} \\
& <2 n^{1 / p} \log n, n \geqq 10 .
\end{aligned}
$$

Lemma 13. Given an arbitrary fixed number $K<\infty$, and fixed $\varepsilon$ and $\lambda, 0<\varepsilon \leqq 1,0<\lambda<p-1$, where $1<p<\infty$, then

$$
K\left\{\frac{m^{1 / p} \log m}{n}+\frac{n^{1 / p} \log n}{m}\right\}=o(1), m, n \rightarrow \infty,
$$

provided that $n / m \leqq \varepsilon^{-1} m^{\lambda}$ and $m / n \leqq \varepsilon^{-1} n^{\lambda}$ as $m, n \rightarrow \infty$.

Proof. Again we satisfy ourselves by proving that under these restrictions, $K\left(m^{1 / p} \log m\right) / n=o(1), m, n \rightarrow \infty$, the proof of the other part being the same. But then $m<\varepsilon^{-1} n^{1+\lambda}$, and so, taking $\varepsilon=1$ for convenience,

$$
\begin{aligned}
K \frac{m^{1 / p} \log m}{n} & <K \frac{n^{1 / p+\lambda / p} \log n^{1+\lambda}}{n} \\
= & K(1+\lambda) \frac{\log n}{n^{1-1 / p-\lambda / p}} \\
& =o(1), n \rightarrow \infty
\end{aligned}
$$

provided that $\lambda<p-1$.

Proof of the main results. By the earlier remarks, to establish the conditions under which the sequence $\left\{h_{m n}\right\}$ will exhibit the principle of localization, it is necessary and sufficient to establish the conditions under which the sequence $\left\{\beta_{m n}\right\}$ is a null sequence. By (1) and (3),

$$
\begin{aligned}
\beta_{m n}= & \frac{1}{4 \pi^{2}} \sum_{0,0}^{m, n}\left(\begin{array}{c}
m \\
k
\end{array}\right)\left(\begin{array}{c}
n \\
l
\end{array}\right) \int_{E(\dot{\theta})} f(s, t) \frac{\sin (k+1 / 2) s}{\sin s / 2} \frac{\sin (l+1 / 2) t}{\sin t / 2} d s d t \\
& \times \int_{0,0}^{1,1} u^{k}(1-u)^{m-k} v^{l}(1-v)^{n-l} d g(u, v) .
\end{aligned}
$$

Interchanging the order of summation and integration, which is permissible in this case since the sum is finite, and applying Lemma. 1 , we get 


$$
\begin{aligned}
\beta_{m n}= & \frac{1}{4 \pi^{2}} \int_{E(\delta)} f(s, t) d s d t \int_{0,0}^{1,1} \rho_{1}^{m} \rho_{2}^{n} \phi(m ; \alpha, s) \phi(n ; \beta, t) d g(u, v) \\
= & \frac{1}{4 \pi^{2}}\left\{\int_{\delta,-\delta}^{\pi, \delta}+\int_{-\pi,-\delta}^{-\delta, \delta}+\int_{-\delta, \delta}^{\delta, \pi}+\int_{-\delta,-\pi}^{\delta,-\delta}\right\} f(s, t) d s d t \\
& \times \int_{0,0}^{1,1} \rho_{1}^{m} \rho_{2}^{n} \phi(m ; \alpha, s) \phi(n ; \beta, t) d g(u, v) \\
= & \lambda_{m n}^{1}+\lambda_{m n}^{2}+\lambda_{m n}^{3}+\lambda_{m n}^{4} .
\end{aligned}
$$

We assume that the mass points of $d g(u, v)$ are all bounded uniformly from the boundary of the unit square, and that $\tau$ is small enough so that $\theta(\tau)$ contains no mass points of $d g(u, v)$. We also assume that $g(u, v)$ satisfies a Lipschitz condition of order one and Lipschitz constant $M$ in every simply connected region of $\theta(\tau)$ over which $g(u, v)$ is continuous, and we let $\sigma$ denote the union of all such regions. Finally, we assume that in $\theta(\tau), g(u, v)$ has finitely many lines of discontinuity, $u=u_{i}, i=1,2, \cdots, k, v=v_{j}, j=1,2, \cdots, l$, such that $\tau \leqq u_{i}, v_{j} \leqq 1-\tau$ for all $i, j$, and that the difference functions $\left\{g_{i}(v), g_{j}(u)\right\}$, where $g_{i}(v)=g\left(u_{i}^{+}, v\right)-g\left(u_{i}^{-}, v\right)$ and

$$
g_{j}(u)=g\left(u, v_{j}^{+}\right)-g\left(u, v_{j}^{-}\right),
$$

all satisfy a Lipschitz condition of order one and Lipschitz constant $M$ on $\theta(\tau)$. We denote the set of these lines by $\omega$.

Thus, $\lambda_{m n}^{i}, i=1,2,3,4$, has three components, namely, due to integration over $\Delta(\tau)$, then due to integration over $\sigma$, and finally due to integration over $\omega$. We denote these components by $\delta_{m n}^{i}, \sigma_{m n}^{i}$ and $\omega_{m n}^{i}$, so that

$$
\lambda_{m n}^{i}=\delta_{m n}^{i}+\sigma_{m n}^{i}+\omega_{m n}^{i}, \quad i=1,2,3,4,
$$

and by (4), (5) and (6), we have

$$
\begin{aligned}
\delta_{m n} & =\delta_{m n}^{1}+\delta_{m n}^{2}+\delta_{m n}^{3}+\delta_{m n}^{4} \\
\sigma_{m n} & =\sigma_{m n}^{1}+\sigma_{m n}^{2}+\sigma_{m n}^{3}+\sigma_{m n}^{4} \\
\omega_{m n} & =\omega_{m n}^{1}+\omega_{m n}^{2}+\omega_{m n}^{3}+\omega_{m n}^{4}
\end{aligned}
$$

Proof of Theorem 1. From (11) and (12), we get

$$
\begin{aligned}
\left|\delta_{m n}^{1}\right| & =\frac{1}{4 \pi^{2}}\left|\int_{\delta,-\delta}^{\pi, \delta} f(s, t) d s d t \int_{\Delta(\tau)} \rho_{1}^{m} \rho_{2}^{n} \phi(m ; \alpha, s) \phi(n ; \beta, t) d g(u, v)\right| \\
& \leqq \frac{1}{4 \pi^{2}} \int_{\delta,-\delta}^{\pi, \delta}|f(s, t)| d s d t \int_{\Delta(\tau)} \rho_{1}^{m} \rho_{2}^{n}|\phi(m ; \alpha, s)||\phi(n ; \beta, t)||d g(u, v)| \\
& \leqq \frac{k n \gamma}{\pi^{2}} e^{-m \tau(1-\tau) \dot{\delta}^{2} / 12} V(g) \int_{\delta,-\delta}^{\pi, \delta} e^{-n \tau(1-\tau) t^{2} / 12}|f(s, t)| d s d t \\
& =K_{1} n e^{-\kappa m} I_{1}(n)
\end{aligned}
$$


where we have applied Lemmas 3,6 and 8 , and set $\kappa=\tau(1-\tau) \delta^{2} / 12$, $K_{1}=k \gamma V(g) / \pi^{2}$, where $V(g)$ denotes the total variation of $g(u, v)$ in the unit square, and

$$
I_{1}(n)=\int_{\delta^{\prime}-\delta}^{\pi, \delta} e^{-n \tau(1-\tau) t^{2} / 12}|f(s, t)| d s d t,
$$

and used the observation that in $\Delta(\tau), \rho_{2}^{n}$ is bounded by $e^{-n \tau(1-\tau) t^{2} / 12}$. Now $e^{-n \tau(1-\tau) t^{2} / 12}|f(s, t)| \downarrow 0, n \rightarrow \infty$, except on a set of measure zero, so that $I_{1}(n) \downarrow 0, n \rightarrow \infty$.

That $\left|\delta_{m n}^{2}\right| \leqq K_{2} n e^{-\kappa m} I_{2}(n)$, where $K_{2}$ and $I_{2}(n)$ have the corresponding relation to the rectangle $[-\delta, \delta ;-\pi,-\delta]$, is proved in the same manner. To show that $\left|\delta_{m n}^{3}\right| \leqq K_{3} m e^{-\kappa n} I_{3}(m)$ and $\left|\delta_{m n}^{4}\right| \leqq K_{4} m e^{-\kappa n} I_{4}(m)$, we interchange the roles of $s$ and $t$, and thus of $m$ and $n$. Here, $\kappa$ has the same meaning throughout, and the constants $K_{i}, i=1,2,3,4$, are all finite. Thus

$$
\begin{aligned}
\left|\delta_{m n}\right| & \leqq\left|\delta_{m n}^{1}\right|+\left|\delta_{m n}^{2}\right|+\left|\delta_{m n}^{3}\right|+\left|\delta_{m n}^{4}\right| \\
& \leqq K\left\{n e^{-\kappa m}\left(I_{1}(n)+I_{2}(n)\right)+m e^{-\kappa n}\left(I_{3}(m)+I_{4}(m)\right)\right\}
\end{aligned}
$$

where $K=\max \left\{K_{1}, K_{2}, K_{3}, K_{4}\right\}$. By the same argument as used to prove that $I_{1}(n) \downarrow 0, n \rightarrow \infty$, we have that $I_{2}(n) \downarrow 0, n \rightarrow \infty$, and $\left(I_{3}(m)+I_{4}(m)\right) \downarrow 0, m \rightarrow \infty$. Applying this to the right hand side of (14), we get $\delta_{m n}=o(1), m, n \rightarrow \infty$, provided that $m \leqq \varepsilon^{-1} e^{\kappa n}$ and $n \leqq \varepsilon^{-1} e^{\kappa m}$ as $m, n \rightarrow \infty$. This completes the proof of the theorem.

Proof of Theorem 2. Taking $\tau=1 / 2$, we may assume that the Lebesgue measure of $\sigma$ is unity, that is, the measure of $[1,1 ; 0,0] \sim \sigma$ is zero. As before, we have

$$
\left|\sigma_{m n}^{1}\right| \leqq \frac{1}{4 \pi^{2}} \int_{\tilde{\delta},-\tilde{\delta}}^{\pi, \tilde{\delta}}|f(s, t)| d s d t \int_{\sigma} \rho_{1}^{m} \rho_{2}^{n}|\phi(m ; \alpha, s)||\phi(n ; \beta, t) \| d g(u, v)|,
$$

and since on $\sigma$ the measure $|d g(u, v)|$ is majorized by $M d u d v$, we have by Lemmas 3 and 7 ,

$$
\begin{aligned}
\left|\sigma_{m n}^{1}\right| \leqq & \frac{M \gamma}{2 \pi^{2}} \int_{\delta,-\delta}^{\pi, \delta}|f(s, t)| d s d t \int_{0,0}^{1,1} \rho_{1}^{m} \frac{\sin n t v}{t} \mid d u d v \\
& +\frac{M \gamma}{4 \pi^{2}} \int_{\delta,-\delta}^{\pi, \delta}|f(s, t)| d s d t \int_{0,0}^{1,1} \rho_{1}^{m}|\psi(n ; t, v)| d u d v .
\end{aligned}
$$

Now

$$
\begin{aligned}
\int_{0,0}^{1,1} \rho_{1}^{m}|\psi(n ; t, v)| d u d v & =\int_{0}^{1} \rho_{1}^{m} d u \int_{0}^{1}|\psi(n ; t, v)| d v \\
& \leqq \delta^{\prime \prime} c m^{-1}
\end{aligned}
$$


uniformly in $t, m$ and $n, 0<\delta \leqq s \leqq \pi$, by Lemmas 7 and 10. Thus the last term on the right in (15) is bounded by $\left(M \gamma I c \delta^{\prime \prime}\right) /\left(4 \pi^{2} m\right)=o(1)$, $m \rightarrow \infty$, where we have set

$$
I=\int_{\delta,-\delta}^{\pi, \delta}|f(s, t)| d s d t
$$

Next, applying Lemma 10 and changing the order of integration in the first term on the right in (15), we get

$$
\begin{aligned}
\left|\sigma_{m n}^{1}\right| & \leqq \frac{M \gamma c}{2 \pi^{2} m} \int_{0}^{1}\left\{\int_{\delta,-\delta}^{\pi, \delta}|f(s, t)|\left|\frac{\sin n t v}{t}\right| d s d t\right\} d v \\
& +o(1), m \rightarrow \infty .
\end{aligned}
$$

Now suppose that $f(s, t) \in B(N(\delta))$, that is, $|f(s, t)| \leqq B<\infty$ almost everywhere in $N(\delta)$. Then

$$
\begin{aligned}
\left|\sigma_{m n}^{1}\right| \leqq & \frac{B M \gamma c \pi}{2 \pi^{2} m} \int_{0}^{1}\left\{\int_{-\delta}^{\delta}\left|\frac{\sin n t v}{t}\right| d t\right\} d v+o(1) \\
= & \frac{B M \gamma c}{\pi m} \int_{0}^{1}\left\{\int_{0}^{\delta}\left|\frac{\sin n t v}{t}\right| d t\right\} d v+o(1) \\
& <2 \frac{B M \gamma c}{\pi m} \log n+o(1) \\
= & K \frac{\log n}{m}+o(1), m \rightarrow \infty, n \geqq 10
\end{aligned}
$$

by Lemma 11. Thus $\sigma_{m n}^{1}=o(1), m, n \rightarrow \infty$, provided that for some fixed but otherwise arbitrary $\varepsilon$ and $\lambda, 0<\varepsilon \leqq 1,0<\lambda<1$, we have $n \leqq \varepsilon^{-1} e^{m^{\lambda}}$ as $m, n \rightarrow \infty$.

That $\sigma_{m n}^{2}=o(1), m, n \rightarrow \infty ; n \leqq \varepsilon^{-1} e^{m^{\lambda}}$, is proved in a similar manner. To prove that $\sigma_{m n}^{3}+\sigma_{m n}^{4}=o(1), m, n \rightarrow \infty, m \leqq \varepsilon^{-1} e^{n^{\lambda}}$, we again reverse the roles of $s$ and $t$, and so of $m$ and $n$. This completes the proof of Theorem 2 for the case where $f(s, t) \in B(N(\delta))$.

If $f(s, t) \in L_{p}(N(\delta)), 1<p<\infty$, then in (16),

$$
\begin{aligned}
\int_{\delta,-\delta}^{\pi, \delta} & |\hat{f}(s, t)|\left|\frac{\sin n t v}{t}\right| d s d t \\
\leqq & \left\{\int_{\delta,-\delta}^{\pi, \delta}|f(s, t)|^{p} d s d t\right\}^{1 / p}\left\{\int_{\delta,-\delta}^{\pi, \delta}\left|\frac{\sin n t v}{t}\right|{ }^{q} d s d t\right\}^{1 / q} \\
& <2 \operatorname{In}^{1 / p} \log n \\
= & K^{\prime} n^{1 / p} \log n, n \geqq 10,
\end{aligned}
$$

where we equated the first integral to $I$, and applied Lemma 12 to the second integral. Then by (16), 


$$
\begin{aligned}
\left|\sigma_{m n}^{1}\right| & \leqq \frac{K^{\prime} M \gamma c}{2 \pi^{2}} \cdot \frac{n^{1 / p} \log n}{m}+o(1) \\
& \leqq K_{1} \frac{n^{1 / p} \log n}{m}, m \rightarrow \infty, n \geqq 10,
\end{aligned}
$$

the $o(1)$ term being absorbed in the first term.

Proceeding as in the first part of the proof with respect to $\sigma_{m n}^{i}$, $i=2,3,4$, and combining the results, we get

$$
\begin{aligned}
\left|\sigma_{m n}\right| & \leqq K\left\{\frac{n^{1 / p} \log n}{m}+\frac{m^{1 / p} \log m}{n}\right\}, m, n \geqq 10 \\
& =o(1), m, n \rightarrow \infty
\end{aligned}
$$

by Lemma 13 , provided that $m / n \leqq \varepsilon^{-1} n^{\lambda}$ and $n / m \leqq \varepsilon^{-1} m^{\lambda}$, or equivalently provided that $\varepsilon m^{-\lambda} \leqq m / n \leqq \varepsilon^{-1} n^{\lambda}, 0<\varepsilon \leqq 1,0<\lambda<p-1$. This completes the proof of Theorem 2 .

Proof of Theorem 3. Let $\omega_{m n}^{1, i}$ be the contribution to $\omega_{m n}^{1}$ due to integration along the line $u=u_{i}$, and let $\omega_{m n}^{1, j}$ be the contribution to $\omega_{m n}^{1}$ due to integration along the line $v=v_{j}$. Then

$$
\omega_{m n}^{1, i}=\frac{1}{4 \pi^{2}} \int_{\delta,-\delta}^{\pi, \delta} f(s, t) d s d t \int_{u_{i}^{-}, 0}^{u_{i}^{+}, 1} \rho_{1}^{m} \rho_{2}^{n} \phi(m ; \alpha, s) \phi(n ; \beta, t) d g(u, v) .
$$

By Lemma $6, \rho_{1}^{m} \leqq e^{-\kappa m}$, where again $\kappa=\tau(1-\tau) \delta^{2} / 12$. Then by Lemmas 3 and 8 ,

$$
\begin{aligned}
\left|\omega_{m n}^{1, i}\right| & \leqq \frac{M n \gamma e^{-\kappa m}}{\pi^{2}} \int_{\delta,-\delta}^{\pi, \delta}|f(s, t)| d s d t \\
& =\frac{M \gamma I_{1}}{\pi^{2}} n e^{-\kappa m} \\
& =K_{1}^{\prime} n e^{-\kappa m}
\end{aligned}
$$

where $I_{1}$ and $K_{1}^{\prime}$ have the obvious meanings, $K_{1}^{\prime}$ being an absolute constant independent of $i$. Since there are at most $k<\infty$ such lines, the total contribution of these $k$ lines to $\omega_{m n}^{1}$ does not exceed

$$
k K_{1}^{\prime} n e^{-\kappa m}=K^{\prime} n e^{-\kappa m} .
$$

Next, we have

$$
\omega_{m n}^{1, j}=\frac{1}{4 \pi^{2}} \int_{\delta,-\delta}^{\pi, \delta} f(s, t) d s d t \int_{0, v_{j}^{-}}^{1, v_{j}^{+}} \rho_{1}^{m} \rho_{2}^{n} \phi(m ; \alpha, s) \dot{\phi}(n ; \beta, t) d g(u, v),
$$

and so, by Lemmas 3,6 and 8 , 


$$
\begin{aligned}
\left|\omega_{m n}^{1, j}\right| \leqq & \frac{k n \gamma M}{\pi^{2}} \int_{\delta,-\delta}^{\pi, \delta}|f(s, t)| e^{-n \tau(1-\tau) t^{2} / 12} d s d t \int_{0}^{1} \rho_{1}^{m} d u \\
& <\frac{k \gamma M c}{\pi^{2}} \frac{n}{m} I_{1}(n) \\
= & K_{1}^{\prime \prime} \frac{n}{m} I_{1}(n)
\end{aligned}
$$

where $k$ now is the constant of Lemma 8 , and $I_{1}(n)$ is the same as in the proof of Theorem 1. Here again $K_{1}^{\prime \prime}$ is an absolute constant independent of $j$, and $I_{1}(n) \downarrow 0, n \rightarrow \infty$. Since there are at most $l<\infty$ such lines, the total contribution of these to $\omega_{m n}^{2}$ is bounded absolutely by

$$
l K_{1}^{\prime \prime} \frac{n}{m} I_{1}(n)=K^{\prime \prime} \frac{n}{m} I_{1}(n)
$$

Thus by (18) and (20),

$$
\begin{aligned}
\left|\omega_{m n}^{1}\right| & \leqq K^{\prime} n e^{-<m}+K^{\prime \prime} \frac{n}{m} I_{1}(n) \\
& \leqq K_{1}\left(n e^{-\kappa m}+\frac{n}{m} I_{1}(n)\right)
\end{aligned}
$$

By similar reasoning, we get

$$
\begin{aligned}
& \left|\omega_{m n}^{2}\right| \leqq K_{2}\left(n e^{-\kappa m}+\frac{n}{m} I_{2}(n)\right) . \\
& \left|\omega_{m n}^{3}\right| \leqq K_{3}\left(m e^{-\kappa n}+\frac{m}{n} I_{3}(m)\right) \\
& \left|\omega_{m n}^{4}\right| \leqq K_{4}\left(m e^{-\kappa n}+\frac{m}{n} I_{4}(m)\right)
\end{aligned}
$$

Taking $K=2 . \max \left\{K_{1}, K_{2}, K_{3}, K_{4}\right\}$ and combining the results, we have

$$
\begin{aligned}
\left|\omega_{m n}\right| \leqq & K\left\{m e^{-\kappa n}+n e^{-\kappa m}\right\} \\
& +K\left\{\frac{n}{m}\left(I_{1}(n)+I_{2}(n)\right)+\frac{m}{n}\left(I_{3}(m)+I_{4}(m)\right)\right\} \\
= & o(1), m, n \rightarrow 0 . \varepsilon \leqq m / n \leqq \varepsilon^{-1},
\end{aligned}
$$

where the first part goes to zero by Lemma 9, and the second part because of the convergence of the integrals $I_{1}(n), I_{2}(n), I_{3}(m)$ and $I_{4}(m)$ to zero. This completes the proof for $f(s, t) \in L[\pi, \pi ;-\pi,-\pi]$.

If $f(s, t) \in L_{p}(N(\delta)), 1<p<\infty$, then 


$$
\begin{aligned}
\left|\omega_{m n}^{1, i}\right| \leqq & \frac{M \gamma e^{-\kappa m}}{4 \pi^{2}} \int_{\delta,-\delta}^{\pi, \delta}|f(s, t)| d s d t \int_{0}^{1}\left|\rho_{2}^{n} \phi(n ; \beta, t)\right| d v \\
\leqq & \frac{M \gamma e^{-\kappa m}}{2 \pi^{2}} \int_{0}^{1}\left\{\int_{\delta,-\delta}^{\pi, \delta}|f(s, t)|\left|\frac{\sin n t v}{t}\right| d s d t\right\} d v \\
& +\frac{M \gamma e^{-\kappa m}}{4 \pi^{2}} \int_{\delta,-\delta}^{\pi, \delta}|f(s, t)| d s d t \int_{0}^{1}|\psi(n ; t, v)| d v
\end{aligned}
$$

by Lemma 7. By (17), the integral in brackets in the first term on the right is bounded by $K_{1}^{\prime} n^{1 / p} \log n$. Thus the first term on the right is bounded $M \gamma K_{1}^{\prime} e^{-\kappa m} n^{1 / p} \log n / 2 \pi^{2}$. Since $\psi(n ; t, v)$ is bounded absolutely and uniformly, the second term is $o(1)$ as $m \rightarrow \infty$. Since there are at most $k$ such lines, their total contribution to $\omega_{m n}^{1}$ is bounded by

(21) $\frac{k M \gamma K_{1}^{\prime}}{2 \pi^{2}} e^{-\kappa m} n^{1 / p} \log n+k \cdot o(1)=K^{\prime} e^{-\kappa m} n^{1 / p} \log n, m \rightarrow \infty$.

On the other hand, by (19),

$$
\begin{aligned}
\left|\omega_{m n}^{1, j}\right| \leqq & \frac{\gamma M c}{2 \pi^{2} m} \int_{\delta,-\delta}^{\pi, \delta}|f(s, t)|\left|\frac{\sin n t v_{j}}{t}\right| d s d t \\
& +\frac{\gamma M c}{4 \pi^{2} m} \int_{\delta,-\delta}^{\pi, \delta}\left|f(s, t) \| \psi\left(n ; t, v_{j}\right)\right| d s d t \\
= & \frac{\gamma M c K_{1}^{\prime}}{2 \pi^{2}} \frac{n^{1 / p} \log n}{m}+o(1), m \rightarrow \infty \\
= & K_{1}^{\prime \prime} \frac{n^{1 / p} \log n}{m}+o(1), m \rightarrow \infty .
\end{aligned}
$$

The contribution of finitely many, say $l$, such lines is then bounded by

$$
l K_{1}^{\prime \prime} \frac{n^{1 / p} \log n}{m}+l \cdot o(1)=K^{\prime \prime} \frac{n^{1 / p} \log n}{m}, m \rightarrow \infty,
$$

the $o(1)$ term being absorbed in the first term. Combining (21) and (22), and setting $K_{1}=\max \left\{K^{\prime}, K^{\prime \prime}\right\}$, we have

$$
\begin{aligned}
\left|\omega_{m n}^{1}\right| & \leqq K_{1} n^{1 / p} \log n\left\{e^{-\kappa m}+\frac{1}{m}\right\}, m \rightarrow \infty \\
& =o(1), m, n \rightarrow \infty, n / m \leqq \varepsilon^{-1} m^{\lambda}, 0<\lambda<p-1
\end{aligned}
$$

by Lemma 13, since for $m$ sufficiently large, $e^{-\kappa m}<m^{-1}$.

Proceeding as before with regard to $\omega_{m n}^{i}, i=2,3,4$, and combining the results, we get

$$
\omega_{m n}=o(1), m, n \rightarrow \infty, \varepsilon m^{-\lambda} \leqq m / n \leqq \varepsilon^{-1} n^{\lambda},
$$


where $0<\lambda<p-1$ is arbitrary but fixed.

If $f(s, t) \in B(N(\delta))$, then a similar calculation yields the result

$$
\omega_{m n}=o(1), m, n \rightarrow \infty, m \leqq \varepsilon^{-1} e^{n^{\lambda}}, n \leqq \varepsilon^{-1} e^{m^{\lambda}},
$$

where $\varepsilon$ and $\lambda$ are arbitrary but fixed, $0<\varepsilon \leqq 1$, and now $0<\lambda<1$. Since the calculations are rather obvious, we avoid the details. This completes the proof of Theorem 3 .

\section{REFERENCES}

1. J. A. Clarkson, and C. R. Adams, On definitions of bounded variation for functions of two variables, Trans. Amer. Math. Soc., 35 (1933), 824-854.

2. F. Hallenbach, Zur Theorie der Limitierungsverfahren von Doppelfolgen, InaugeralDissertation, Rheinische Friedrich-Wilhelms-Universität, Bonn (1933).

3. O. Szász, Gibbs phenomenon for Hausdorff means, Trans. Amer. Math. Soc., 69 (1950), 440-456.

4. L. Tonelli, Sulla convergenza delle serie doppie di Fourier, Ann. di Mat. Pura ed Appl., 4 (1927), 29-72.

5. F. Ustina, Convergence of Double Fourier Series, Ann. di Mat. Pura ed Appl., 85 (1970), 21-48.

6. A. Zygmund, Trigonometric Series, Vol. I and II. University Press, Cambridge (1959).

Received May 22, 1970, and in revised form September 21, 1970. The preparation of this paper was financed, in part, by a Canadian Mathematical Congress Summer Research Grant (1969).

University of AlBerta 



\section{PACIFIC JOURNAL OF MATHEMATICS}

\section{EDITORS}

H. SAMELSON

Stanford University

Stanford, California 94305

C. R. Новву

University of Washington

Seattle, Washington 98105
J. DUGundJI

Department of Mathematics

University of Southern California

Los Angeles, California 90007

RICHARD ARENS

University of California

Los Angeles, California 90024

\section{ASSOCIATE EDITORS}
E. F. BECKENBACH
B. H. NeumanN
F. WOLF
K. YOSHIDA

\section{SUPPORTING INSTITUTIONS}
UNIVERSITY OF BRITISH COLUMBIA
CALIFORNIA INSTITUTE OF TECHNOLOGY
UNIVERSITY OF CALIFORNIA
MONTANA STATE UNIVERSITY
UNIVERSITY OF NEVADA
NEW MEXICO STATE UNIVERSITY
OREGON STATE UNIVERSITY
UNIVERSITY OF OREGON
OSAKA UNIVERSITY
UNIVERSITY OF SOUTHERN CALIFORNIA
STANFORD UNIVERSITY
UNIVERSITY OF TOKYO
UNIVERSITY OF UTAH
WASHINGTON STATE UNIVERSITY
UNIVERSITY OF WASHINGTON
AMERICAN MATHEMATICAL SOCIETY CHEVRON RESEARCH CORPORATION TRW SYSTEMS
NAVAL WEAPONS CENTER 


\section{Pacific Journal of Mathematics}

\section{Vol. 37, No. $1 \quad$ January, 1971}

Gregory Frank Bachelis and Haskell Paul Rosenthal, On unconditionally

converging series and biorthogonal systems in a Banach space .........

Richard William Beals, On spectral theory and scattering for elliptic

operators with singular potentials .........................

J. Lennart (John) Berggren, Solvable and supersolvable groups in which every element is conjugate to its inverse ........................ 21

Lindsay Nathan Childs, On covering spaces and Galois extensions ..........

William Jay Davis, David William Dean and Ivan Singer, Multipliers and

unconditional convergence of biorthogonal expansions..............

Leroy John Derr, Triangular matrices with the isoclinal property ............

Paul Erdős, Robert James McEliece and Herbert Taylor, Ramsey bounds for

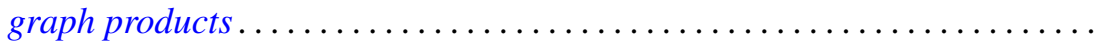

Edward Graham Evans, Jr., On epimorphisms to finitely generated

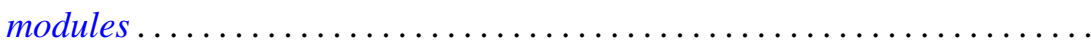

Hector O. Fattorini, The abstract Goursat problem ................. 51

Robert Dutton Fray and David Paul Roselle, Weighted lattice paths .........

Thomas L. Goulding and Augusto H. Ortiz, Structure of semiprime $(p, q)$

radicals ...........................................

E. W. Johnson and J. P. Lediaev, Structure of Noether lattices with join-principal maximal elements ....

David Samuel Kinderlehrer, The regularity of minimal surfaces defined over

slit domains

Alistair H. Lachlan, The transcendental rank of a theory. .

Frank David Lesley, Differentiability of minimal surfaces at the boundary ...

Wolfgang Liebert, Characterization of the endomorphism rings of divisible torsion modules and reduced complete torsion-free modules over complete discrete valuation rings....

Lawrence Carlton Moore, Strictly increasing Riesz norms.

Raymond Moos Redheffer, An inequality for the Hilbert transform ...

James Ted Rogers Jr., Mapping solenoids onto strongly self-entwined,

circle-like continua..........................

Sherman K. Stein, B-sets and planar maps ................... 217

Darrell R. Turnidge, Torsion theories and rings of quotients of Morita

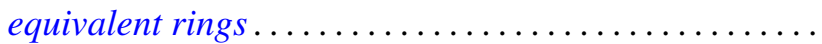

Fred Ustina, The Hausdorff means of double Fourier series and the principle of localization ................................

Stanley Joseph Wertheimer, Quasi-compactness and decompositions for arbitrary relations.

Howard Henry Wicke and John Mays Worrell Jr., On the open continuous images of paracompact $\check{C}$ ech complete spaces... 